\title{
Myeloid Neoplasms with Germline Predisposition Associated with Inherited Bone Marrow Failure Syndromes
}

National Cancer Institute

\section{Source}

National Cancer Institute. Myeloid Neoplasms with Germline Predisposition Associated with Inherited Bone Marrow Failure Syndromes. NCI Thesaurus. Code C151922.

Familial myelodysplastic syndromes/ acute myeloid leukemias associated with inherited bone marrow failure syndromes. 